\title{
What causes grief in dementia caregivers?
}

\author{
Katarzyna Warchol-Biedermann ${ }^{\text {a }}$, Ewa Mojs ${ }^{\text {a }}$, Rikke Gregersen ${ }^{\text {b }}$, Kirsten Maibom ${ }^{\text {, }}$, \\ José C. Millán-Calenti ${ }^{\mathrm{c}, \mathrm{d}}$, Ana Maseda ${ }^{\mathrm{c}, \mathrm{d}}$ \\ ${ }^{a}$ Department of Clinical Psychology, Poznan University of Medical Sciences, Centrum Stomatologii, ul. Bukowska 70, 60-812 \\ Poznan, Poland \\ ${ }^{\mathrm{b}}$ Centre for Research in Aging and Dementia. Faculty of Health Sciences, VIA University College, 8200 Aarhus N. Denmark \\ ${ }^{\mathrm{c}}$ Research, Development and Innovation Department, Gerontological Complex La Milagrosa, Provincial Association of Pensioners \\ and Retired People from A Coruña, Avenida de Cadiz-5, E-15008 A Coruña, Spain \\ ${ }^{\mathrm{d}}$ Gerontology Research Group, Department of Medicine, Faculty of Health Sciences, Universidade da Coruña, E-15071 A Coruña, \\ Spain
}

\begin{abstract}
Alzheimer's disease (AD) is the most prevalent neurodegenerative disease in the world. Most AD patients become dependent on their relatives, i.e. family caregivers. Providing care to a person with AD influences caregiver's life and leads to feelings of grief, which often precede caregiver depression. The purpose of the article was to evaluate the Meuser and Marwit Caregiver Grief Inventory (MM-CGI-50) for use in Polish family caregivers and to find out determinants of grief of family caregivers of AD individuals living in Poland. A sample of 151 spouse and adult child caregivers of community-dwelling $\mathrm{AD}$ patients (95 females and 56 males) was interviewed to determine the influence of such factors as caregiver's age, gender, family relation to the care recipient (CR) and caregiving-related changes in caregiver's working time, leisure time and material status to find out the impact of caregiving role on intensity of caregiver grief. Caregiver grief was measured by means of MM-CGI-50. Additionally, carers were administered a questionnaire including patient's and caregiver's demographics. Also, CR's dementia assessment was informantbased and determined with investigator-administered clinical dementia rating (CDR) scale. Of all analyzed factors, only caregiver's informant dementia rating significantly influenced caregiver grief. To conclude, the effect of caregiver's age, gender, family relation to the CR and caregiving-related changes in caregiver's working time; leisure time and material status could not be found. To add, MM-CGI-50 can be effectively used to assess grief in Polish family caregivers of $\mathrm{AD}$ patients.
\end{abstract}

Keywords: Caregiving-informal, Dementia Evaluation

\section{Introduction}

$\mathrm{AD}$ is the most prevalent neurodegenerative disease in the world. Current epidemiological estimates indicate in the U.S, for example, that one in eight individuals over 65 years of age will develop AD. What's more, the total number of persons afflicted with AD in the world is expected to exceed 107 million by 2025 (2012 Alzheimer's disease. Facts and Figures, 2012; Grand, Caspar, \& Macdonald, 2011; Grossman et al., 2010; Ozarowski, Kupsz, \& Torlinska, 2006). The symptoms of the disease include not only progressing memory deterioration and cognitive impairment but also depression and behavioral symptoms such as aggression, sleep disturbances or wandering. In the course of the illness most AD patients become dependent on their relatives (spouses, adult children or, much less frequently, members of extended family), i.e. family caregivers.

Providing care to a person with dementia of Alzheimer's type is associated with emotional, physical and financial toll because of its influence on caregiver's lifestyle, professional career and financial status (Adams, 2006; Maslow, 2008). Recently, a number of investigations were devoted to psychosocial problems connected with providing care to a close relation with Alzheimer's. Being a family caregiver has been shown to be a risk factor for depression, anxiety and distress. According to several authors, the onset of caregiver's anxious depression depended on such factors as caregiver's age, gender and relation to the CR (Andrén \& Elmsta ${ }^{\circ}$ hl, 2008; Cuijpers, 2005; de Vugt, Nicolson, Aalten, \& Lousberg, 2005; Epstein-Lubow, Duncan Davis, Miller, \& Tremont, 2008; Mahoney, Regan, Katona, \& Livingston, 2005; 
Melo, Maroco, \& de Mendonca, 2011; Schulz \& Martire, 2004). Observations also indicated that depressive symptoms could be triggered by CR's behavioral disturbances.

Then Meuser and Marwit highlighted the concept of caregiver grief. Grief had initially been defined as a psychological (emotional, cognitive, functional and behavioral responses) response to a loss such as death (Prigerson \& Maciejewski, 2008; Zisook \& Shear, 2009). Meuser and Marwit, however, found out that family caregivers of persons with Alzheimer's disease grieved as they were observing "social" death and intellectual deterioration of their loved ones long time before the actual experience of their death (Marwit \& Meuser, 2002; Meuser \& Marwit, 2001). Meuser and Marwit also developed an inventory to assess caregiver (MM-CGI-50). Based on their analyses, which were carried with the use of MM-CGI-50, they indicated feelings of grief were related to caregiver's perceived personal sacrifice (adverse effect of care on caregiver's professional, social and family life), heartfelt sadness and longing (personal sadness and separation pain), social isolation and diminished perceived social support. Grief in dementia caregivers was distinct from depression and was associated with worse quality of life and mental health morbidity (Kiely, Prigerson, \& Mitchell, 2008; Marwit \& Meuser, 2005; Meuser, Marwit, \& Sanders, 2004; Silverberg, 2008). Some investigators believed that the way an individual carer experienced and coped with caregiving-related grief could influence the quality of care, the process of mourning (grief work) and adjustment after the loved one's death (Gataric, Kinsel, Currie, \& Lawhorne, 2010; Meuser \& Marwit, 2001; Schulz, Boerner, Shear, Zhang, \& Gitlin, 2006; Silverberg, 2007). Caregiver grief reactions would fluctuate with time, were affected by the stage of the disease and could be determined by caregiver's family relation to the CR (Meuser \& Marwit, 2001; Meuser et al., 2004). Simultaneously, it was noted that reaction to these agents could depend on caregiver's sociodemographic characteristics so factors affecting grief in America do not have to play an identical role in other countries (Givens, Prigerson, Kiely, Shaffer, \& Mitchell, 2011; Kiely et al., 2008; Marwit \& Meuser, 2005; McLennon, Bakas, Habermann, \& Meuser, 2014; Meuser \& Marwit, 2001; Meuser et al., 2004). So far, there were only few studies of grief in reaction to dementia caregiving, most of which, were carried out on samples of caregivers living in the American society. Findings from these studies cannot be generalized to caregiver populations living in other countries because of socio- economic and cultural differences between societies, to say the least (McLennon et al., 2014). Consequently, considering both theoretical and practical importance of the impact of grief on dementia caregivers, our aim was to explore agents influencing caregiver grief in a sample of Polish caregivers of individuals with AD with the use of 50item MM-CGI-50. In order to achieve that goal we decided to develop Polish version of that inventory. We wished to find out determinants of caregiver grief so caregiver grief measures in the sample were taken and related to such variables as caregiver's age; gender; family relation to the CR; caregiver's and CR's living arrangements (living together or in separate households); care- giver's informant assessment of CR's level of dementia and caregiving-related changes in caregiver's working time, leisure time and material status.

We hypothesized that these factors would influence grief but we believed their role would not be identical due to relevant socioeconomic and cultural differences between Poland and the U.S.

\section{Method}

One hundred and fifty one current spouse or adult-child caregivers participating in the study were recruited through Day Care and Rehabilitation Center for AD Patients in Poznan (Poland), via a special website designed for the purpose of the study, by an internet advertisement and by word of mouth. Demographics and care-related characteristics of all subjects are presented in Table 1. 


\section{Procedure}

All subjects were interviewed individually and anonymously by the author of the paper either at their homes or in a separate room at day care center facilities. Each participant was informed about the purpose and importance of the study and voluntarily gave their consent to participate. The investigator also made sure subjects knew they could stop the interview at any moment. None of the participants stopped the interview or withdrew from research.

\section{Measures}

Informant dementia assessment: patient's dementia level was determined by way of CDR (Morris, 1993). Six cognitive-functional domains (memory, orientation, judgment and problem solving, community affairs, home and hobbies, and personal affairs) were measured. CDR was carried out as a semistructured interview with the caregiver. The interviewer used scoring rules $(0$ - normal, 0.5 - very mild, $1-$ mild, 2 - moderate, 3 - severe) to determine functional impairment rating and used an algorithm-based composite score as the final rating as suggested by the author of the test (Morris, 1993). In the present study, respondents provided their own assessment of their CR's intellectual and functional impairment. Caregiver's subjective evaluation of CR's functional status was favored because, as MM-CGI-50 authors indicated, it was a more appropriate way to estimate caregiver's grief. It was believed participants' own individual perceptions and assessments of their loved one's health status were more important for their feelings of grief than doctor's diagnosis (Marwit \& Meuser, 2002; Morris, 1993). In CDR, informant impairment rating is thought to be comparable to clinician's rating.

Caregiver grief: following CDR, all subjects filled in validated Polish version of MM-CGI-50 to assess the intensity of caregiver grief (Marwit \& Meuser, 2002).

\section{Validation}

MM-CGI-50 The procedure of validation of MM-CGI-50 consisted of two stages. The first step involved its cross-cultural adaptation while the second step involved determination of test's reliability. First, the questionnaire was translated into Polish by five independent bilingual translators without medical or clinical background about the concept of caregiver grief. Then the translation was synthesized and translated back into English by a professional translator who did not know the original version of MMCGI-50. The resulting translation was then cross-examined with the original English version and discrepancies were corrected. This version was later pilot tested on ten healthy individuals with background in psychology who were native speakers of Polish and fluent in English. They were asked to provide critical feedback on vocabulary used and clarity of test items to ensure questionnaire's crosscultural validity. All participants completed the question-naire within $35 \mathrm{~min}$ and reported that all items were easy to understand and adequate in addressing caregiver grief issues. Then the test was ready for further statistical evaluation of its reliability.

To determine the reliability of MM-CGI-50 the test-retest method was used (Rousson, Gasser, \& Seifert, 2002). First, the test was administered to twenty-five volunteers who were family caregivers of AD patients. After twenty-one days' interval the same group of caregivers was retested again. The total score reliability got an $\mathrm{rs}=0.87$ Spearman-rank correlation coefficient $(\mathrm{p}<0.01)$. The internal consistency was determined by Cronbach's alpha 0.90) and the test-retest reliability analyzed by Cohen's kappa, obtained a range from 0.83 to 0.87 ( $\mathrm{p}<0.01$ ). They specified their level of agreement or disagreement to each statement of the inventory on a symmetric agree-disagree Likert scale [1 - strongly disagree, 2 - disagree, 3 - somewhat agree, 4 - agree, 5 - strongly agree]. Each respondent's score represented their intensity of caregiver grief. 
Table 1

Demographic characteristics of family caregivers and CRs participating in the study

\begin{tabular}{|c|c|}
\hline Statistic & $\mathrm{N}(\%)$ or $\mathrm{M}(\mathrm{SD})$ \\
\hline \multirow[t]{2}{*}{ Age } & 58.9 years $(\mathrm{SD}=10.1)$ \\
\hline & Women $58.2(\mathrm{SD}=10.1)$; men $60.3(\mathrm{SD}=10.1)$ \\
\hline Gender & $56(37 \%)$ men \\
\hline \multicolumn{2}{|l|}{ Marital status } \\
\hline Single & $15(10 \%)$ \\
\hline Widowed & $15(10 \%)$ \\
\hline \multicolumn{2}{|l|}{ Education } \\
\hline Elementary ( 8 years of education completed) & $23(15 \%)$ \\
\hline High school (12 years of education completed) & $67(44 \%)$ \\
\hline College degree (17 years of education completed) & $61(40 \%)$ \\
\hline Caring for wife & $43(28 \%)$ \\
\hline Caring for husband & $41(27 \%)$ \\
\hline Living arrangements & $124(82 \%)$. living together with CR and $27(18 \%)$ living apart \\
\hline \multicolumn{2}{|l|}{ Statistics on CRs } \\
\hline Age & Women $71.4(\mathrm{SD}=6.2)$; men $72.3(\mathrm{SD}=6.7)$ \\
\hline
\end{tabular}

Sociodemographic variables: next, participants of the study completed Caregiver's Questionnaire which asked about their demographic characteristics (age, gender, level of education, current employment and marital status, familial relation to the CR), patient's demographics (age and gender), living arrangements (living together with the CR or in separate households) as well as features of the care situation (caregiving-related changes in caregiver's working time, leisure time and material status).

The results were then collected using Excel spreadsheets so that respondents were divided into the following defined caregiver subgroups: female vs. male caregiver; adult-child vs. spouse caregiver; caregivers living in the same vs. in the separate household; caregivers who devoted to care up to $50 \%$ vs. between $50 \%$ and $100 \%$ of their free time; participants without caregiving-related changes in their working time vs. caregivers who reduced their working time vs. caregivers who quit their jobs; caregivers with vs. caregivers without adverse changes in their material status due to caregiving and participants whose CR had moderate dementia vs. carers of individuals with severe dementia and compare them with the use of appropriate non-parametric statistical tests (Mann-Whitney U test, Kruskal-Wallis test, post hoc Dunn's test). The Correlation between caregiver grief and caregiver's age was determined by Spearman's coefficient.

The design and methods used in the study were approved by the Bioethical Commission of Poznan University of Medical Sciences (Decision No. 10/10).

\section{Results}

A total of 151 family caregivers of patients with a medical diagnosis of dementia of Alzheimer's type which met ICD-10 diagnostic criteria participated in the study. 95 of them $(63 \%)$ were females, who were in $40-83$ age range (mean age $=58.2 ; \mathrm{SD}=10.1)$ while 56 of them $(37 \%)$ were males, who were in 24 75 age range (mean age $=60.3 ; \mathrm{SD}=10.1$ ). The distribution of caregiver grief results is presented in Fig. 1. It is asymmetric and characterized by the following values: M: 156.9; SD: 22.2; Me: 155; $\min .:$ 106; $\max .: 198$. 
Caregiver grief results for both gender groups were characterized by the following statistics: M: 157.4; SD: 21; Me: 155 for females and M: 156.0; SD: 23; Me: 156 for males. The comparison of caregiver grief results in the male and in the female group showed that no statistical differences in caregiver grief values between male and female carers were found (Mann-Whitney U test; $p=0.9233$ ) (see Table 2 for statistical data).

Also, correlation between caregiver grief and caregiver's age could not be found ( $r s=0.008 ; \mathrm{p}<05000$ ). Similarly, caregiver grief did not correlate with age in the male and in the female group taken separately ( $r s=\_0.057 ; p<0.5000$ for men; $r s=0.046 ; p<0.05000$ for women).

All participants of the study provided care to their close relations - mostly mother $(\mathrm{n}=36 ; 24 \%)$, wife (n $=43 ; 28 \%)$, husband $(n=41 ; 27 \%)$ or father $(n=28 ; 19 \%)$. Only $2 \%$ of participants $(n=3)$ cared for distant relatives such as their aunt. Caregiver grief results for adult-child and spousal caregivers were characterized by the following statistics: M: 156.44; SD: 21.66; Me: 154.86 for spouses and M: 157.23; SD: 23; Me: 156 for adult children.

The statistical analysis has not demonstrated that the family relationship (spouse caregiving vs. adultchild caregiving) signifi-cantly influenced caregiver grief values (Mann-Whitney U test; $p=0.7770$ ).

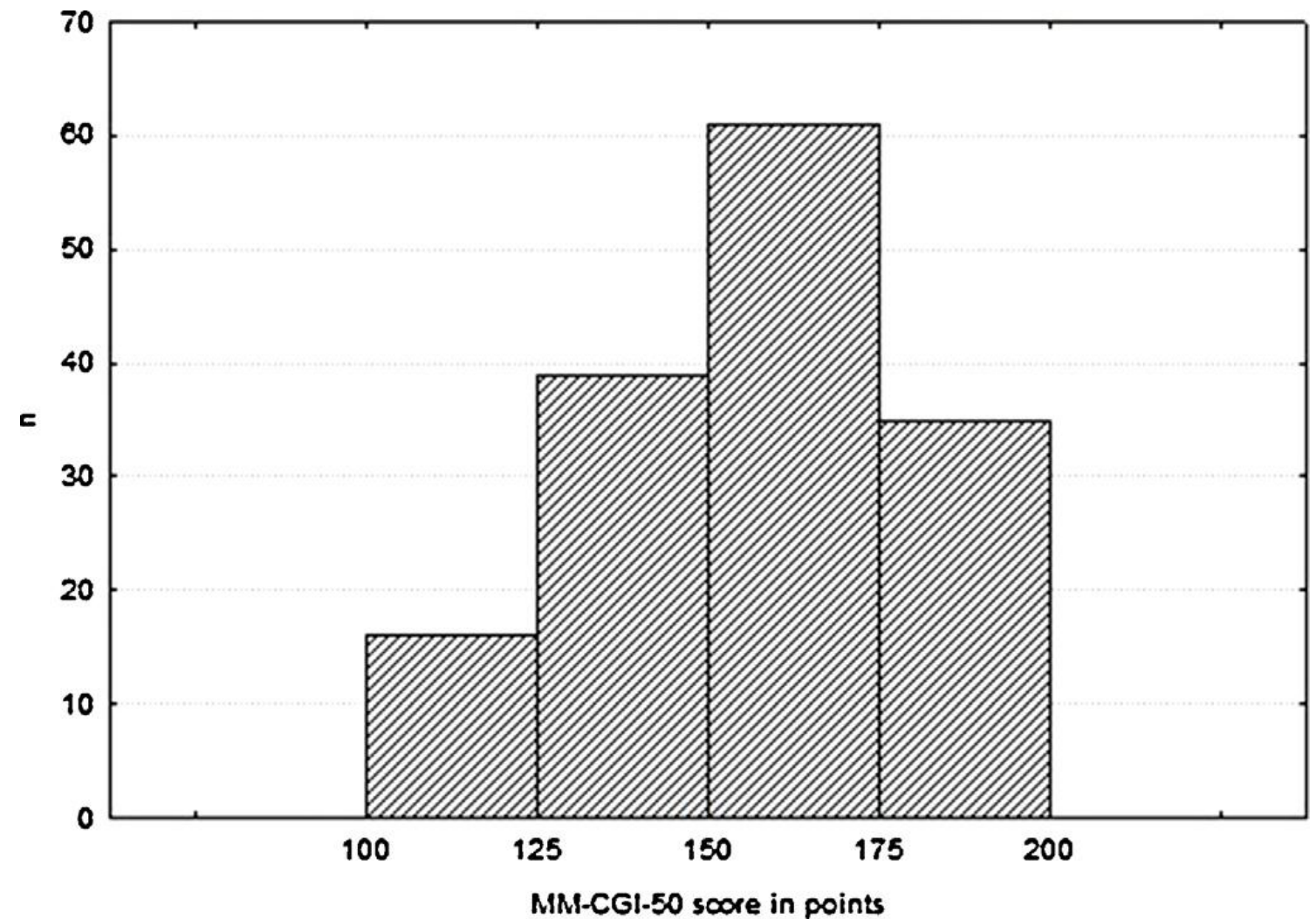

Fig. 1. Distribution of caregiver grief scores in the analyzed sample. 
Table 2

The relation between analyzed factors and caregiver grief scores in caregiver subgroups

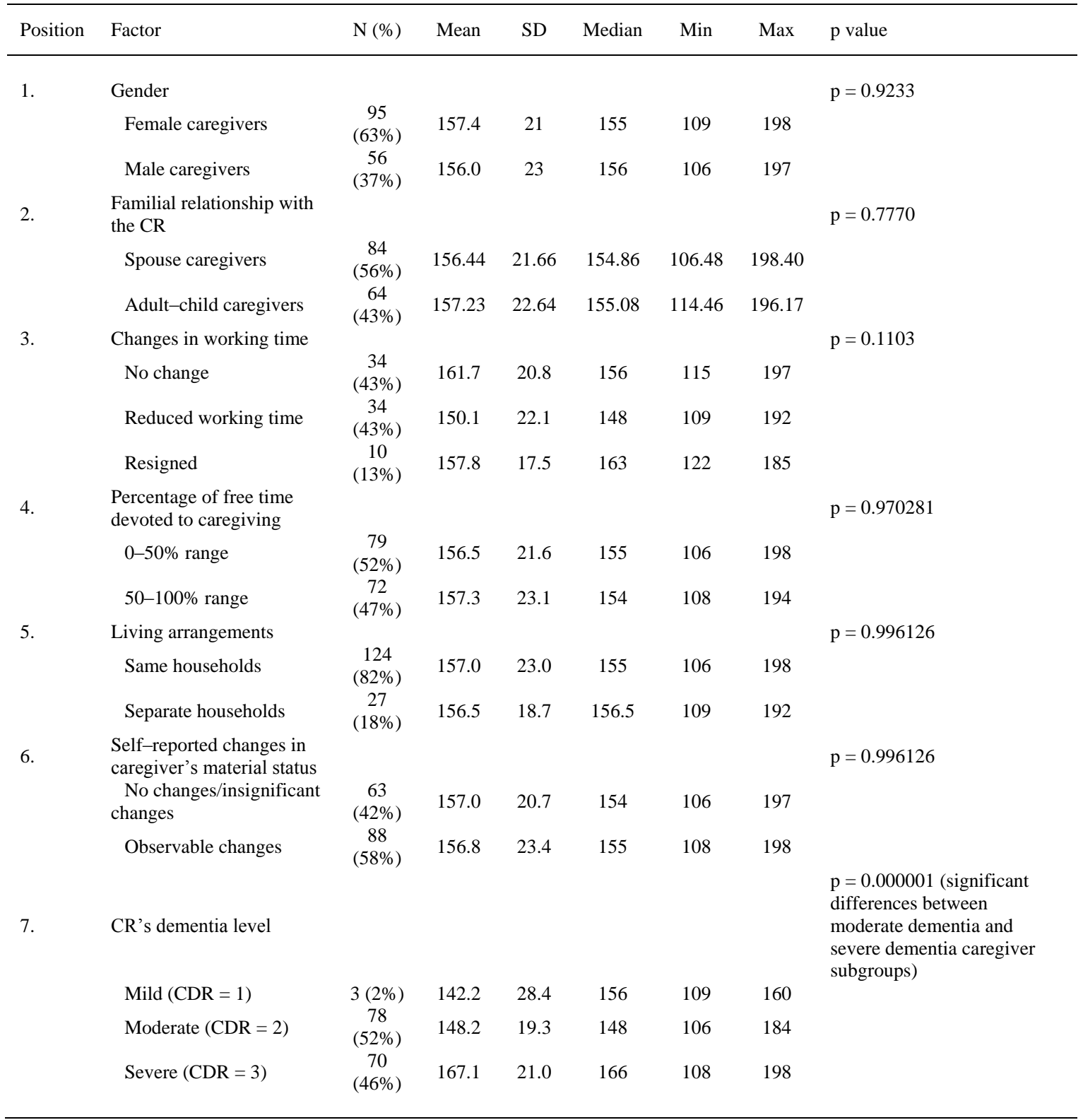

In the investigated sample, 34 (43\%) working age respondents said caregiving did not affect their work while $44(56 \%)$ caregivers reported that caregiving influenced the course of their professional career. To add, 34 (43\%) participants admitted they had to quit extra hours or reduced their working time while 10 $(13 \%)$ of them quit work because of care. Caregiver grief results for these groups of caregivers were characterized by the following statistics: M: 161.7; SD: 20.8; Me: 156 for the "no change" group; M: 150.1; SD: 22.1; Me: 148 for the "reduced working time"' group and M: 157.8; SD: 17.5; Me: 163 for the group of caregivers who quit their jobs. Analysis indicated that there were no statistical differences in caregiver grief between these three groups of caregivers (Kruskal- Wallis test; $p=0.1103$ ).

Additionally, 79 (52\%) participants reported they devoted to care up to $50 \%$ of their free time while 72 of them $(47 \%)$ devoted to care between $50 \%$ and $100 \%$ of their time. These groups of subjects were characterized by the following caregiver grief statistics: M: 156.5; SD: 21.6; Me: 155 for the group of caregivers who to devoted to care up to $50 \%$ of their free time and M: 157.3 ; SD: 23.1; Me: 154 for 
caregivers who devoted to care between $50 \%$ and $100 \%$ of their free time. These groups did not significantly differ in their caregiver grief measures (Mann- Whitney U test; $p=0.970281$ ).

In the examined sample, $124(82 \%)$ of subjects lived in the same household with their CRs while 27 of them $(18 \%)$ lived separately. These groups of subjects were characterized by the following caregiver grief statistics: M: 157; SD: 23; Me: 155 for caregivers living in the same households and M: 156.5; SD: 18.7; Me: 156.5 for caregivers living in separate households. Analysis did not indicate the impact of living arrangements on caregiver grief (Mann-Whitney U test; $\mathrm{p}=0.996126$ ).

Caregivers also evaluated caregiving related changes in their material status. It turned out $88(58 \%)$ of them experienced significant adverse changes in their material status due to caregiving. Caregiver grief results for these groups of caregivers were characterized by the following statistics: M: 157; SD: 20.7; Me: 154 for the "no changes" group and M: 156.8; SD: 23.4; Me: 155 for the group with observable changes in the material status respectively. Here, analysis did not confirm that these changes had any significant impact on caregiver grief (Mann-Whitney $U$ test; $p=0.9849$ ).

Caregiver's informant dementia assessment has shown that $78(52 \%)$ participants provided care for individuals with moderate dementia (CDR: 2), 70 (46\%) cared for individuals with severe dementia (CDR: 3 ) and only 3 of them (2\%) cared for a loved with mild dementia (CDR: 1). Caregiver grief statistics for these groups of participants were the following: M: 142.2; SD: 28.4; Me: 156 for the "mild dementia" caregivers: M: 148.2; SD: 19.3; Me: 148 for "moderate dementia" caregivers and M: 167; SD: 21; Me: 166 for "severe dementia" caregivers respectively. The comparison of caregiver grief scores obtained by the three groups of participants has shown that subjects providing care for patients with severe dementia had significantly greater values of caregiver grief than the group of moderate dementia caregivers (Dunn's post hoc test; $\mathrm{p}=0.000001)$. The group of participants caregiving for mildly demented patients was excluded from statistical analysis because of a small sample size $(n=3)$.

\section{Discussion}

Due to its prevalence and course, $\mathrm{AD}$ has become a serious public health problem. With its characteristic symptoms, AD influences not only patient's life but also the lives of family members who provide care for him or her. As patients' physical and intellectual impairment increases, they lose the ability to function on their own and in daily life activities have to be helped by others, usually their close relatives. The relatives who become family caregivers of persons with AD have to play many roles in their lives: they are usually someone's spouses or partners, parents and professional workers. As they devote more and more time to caregiving, they have to give up many of their private plans and professional career. Additional problems may start when the CR develops behavioral or psychiatric symptoms, which demand special treatment. This problem has been addressed by several papers which pointed to adverse impact of AD caregiving on caregiver's well-being (Cuijpers, 2005; Leo’ n-Salas, Olazarán, Muñiz, GonzálezSalvador, \& Martínez-Martín, 2011; Mahoney et al., 2005; Maslow, 2008; Schulz \& Martire, 2004). Also, observations showed that caring for a relative with dementia may trigger distress and intensified caregiver burden which often result in depression and anxiety (Adams, Smyth, \& McClendon, 2005; EpsteinLubow et al., 2008; Schulz et al., 2008). Recently, studies pointed to caregiver grief which is a universal experience of family caregivers of AD patients (Adams \& Sanders, 2004; Epstein- Lubow et al., 2008; Marwit \& Meuser, 2002; Meuser \& Marwit, 2001; Meuser et al., 2004; Ott, Sanders, \& Kelber, 2007). Caregiver grief was connected not only with burden of the disease and coping with its symptoms but also with a gradual loss of the relationship with the patient who is a caregiver's close family member, isolation and low perceived social support. Some authors suggested intensified grief could be a prelude to complicated grief or depression in susceptible individuals (Frank, 2008; Givens et al., 2011; Marwit \& Meuser, 2005; Peterson, 2006; Zisook \& Shear, 2009). 
This study was devoted to assessment of factors which may affect caregiver grief of family caregivers of AD patients living in Poland. We evaluated the impact of such variables as caregiver's age, gender, living arrangements (living with the CR or not), caregiver's family relation to the CR, caregiver's informant assessment of CR's level of dementia and caregiving-related changes in caregiver's working time, leisure time and material status.

The comparison of caregiver grief scores of subjects who provided care for patients at different levels of dementia has shown that the stage of disease significantly influenced intensity of caregiver grief. To add, caregiver grief scores were the highest among the group of respondents providing care for patients with profound dementia. Similar observations were made by Meuser and Marwit, who conducted their investigation in a similar sample size and indicated that severity of dementia remarkably affected grief scores in their sample of American caregivers (Marwit \& Meuser, 2002, 2005; Meuser \& Marwit, 2001; Meuser et al., 2004). The impact of the stage of dementia on caregiver grief may be related to characteristics of contact with a person with advanced dementia such as poor communication and not being able to share a whole range of thoughts, feelings and experiences. Also, grief may be affected by the fact that caregivers are aware the disease is progressing over time, there is no hope for improvement and their loved ones are approaching the end of their lives. Meuser and Marwit also found that grief depended on family relationship between caregiver and the CR. They observed that the grief process was different for adult-child caregivers than for spouse caregivers and the patterns for each were characteristic at various stages of dementia (Meuser \& Marwit, 2001). Their finding, however, was not confirmed in our current study of Polish family caregivers. These differences may be related to socioeconomic or cultural differences between Poland and the U.S. In Poland, children traditionally maintain close relationship with their parents well into their adulthood. Three-generational families often live together and the emotional ties between elderly parents and their adult children remain strong, both in the urban and in the rural areas.

Furthermore, in our study we did not indicate the significant impact of such factors as caregiver's age, gender or caregiving-related changes in caregiver's working time, leisure time and material status on caregiver grief. Based on these results, we cannot assume these agents do not influence caregiver grief at all but we may suppose their influence is less evident than the effect of CR's dementia status, in relation to which significant differences could be observed.

We indicated that caregiver grief is a feeling, which is connected with family ties and emotional bonds with a relation who has Alzheimer's disease and affected by the stage of disease. Our results have practical implications related to support programs for caregivers. Family members who provide care to their relations with dementia usually receive financial or material support. This type of support is very important but it will not directly influence caregiver grief. Family doctors, nurses, other professionals or volunteers who contact family caregivers should be provided education and training programs to help them understand the phenomenon of caregiver grief and integrate the knowledge about grief into practice so that they will be able to provide adequate emotional support to grieving family caregivers.

\section{Conclusions}

MM-CGI-50 can be effectively used to assess grief in Polish family caregivers of AD patients. Caregiver grief in AD carers living in Poland is associated with their own subjective evaluation of their loved one's dementia level. It can be suggested that factors such as family relation to the CR; caregiving-related changes in caregiver's working time, leisure time and material status do not have a clear impact on caregiver grief in Polish family caregivers. 


\section{Conflict of interest statement}

The authors declare no conflict of interest.

\section{Funding}

The publication was financed by the European Union (grant no. AAL 5/1/2013) Grant for The UnderstAid - a platform that helps informal caregivers to understand and aid their demented relatives, Agreement No. AAL 5/1/2013 (The National Centre for Research and Development Director Decision No. 44/2013 of Feb.20, 2013). The funding bodies were not involved in the study design, in the collection, analysis and interpretation of data; in the writing of the manuscript; and in the decision to submit the manuscript for publication.

\section{Acknowledgements}

The authors would like to thank Prof. Samuel Marwit, Department of Psychology, University of Missouri-St. Louis and Prof. Thomas Meuser, Director of Gerontology at University of Missouri-St. Louis for their agreement to use MM-CGI-50 in our study of Polish caregivers.

\section{References}

Adams, K. B. (2006). The transition to caregiving: Experiences of family members embarking on the dementia caregiving career. Journal of Gerontology: Social Work, 47, 3-30.

Adams, K. B., \& Sanders, S. (2004). Caregiver differences in experience of loss, grief reactions, and depressive symptoms across the stages of Alzheimer's disease: A mixed methods analysis. Dementia: International Journal of Social Research and Practice, 3, 195-210.

Adams, K. B., Smyth, K. A., \& McClendon, M. J. (2005). Psychosocial resources as moderators of the impact of spousal dementia caregiving on depression. Journal of Applied Gerontology, 24, 475-489.

Alzheimers, \& Demen (2012). 2012 Alzheimer's disease. Facts and figures (Vol. 8). Retrieved from http://www.alz.org/downloads/facts figures 2012.pdf Accessed 15.02.13.

Andrén, S., \& Elmsta ${ }^{\circ}$ hl, S. J. (2008). The relationship between caregiver burden, caregivers' perceived health and their sense of coherence in caring for elders with dementia. Journal of Clinical Nursing, 17, 790-799.

Cuijpers, P. (2005). Depressive disorders in caregivers of dementia patients: A system-atic review. Aging \& Mental Health, 9, 325330.

de Vugt, M. E., Nicolson, N. A., Aalten, P., \& Lousberg, R. (2005). Behavioral problems in dementia patients and salivary cortisol patterns in caregivers. Journal of Neuropsy-chiatry and Clinical Neurosciences, 17, 201-207.

Epstein-Lubow, G., Duncan Davis, J., Miller, I. W., \& Tremont, G. (2008). Persisting burden predicts depressive symptoms in dementia caregivers. Journal of Geriatric Psychiatry and Neurology, 21, 198-203.

Frank, J. B. (2008). Evidence for grief as the major barrier faced by Alzheimer caregivers: A qualitative analysis. American Journal of Alzheimers Disease and Other Dementias, 22, 516-527.

Gataric, G., Kinsel, B., Currie, B. G., \& Lawhorne, L. W. (2010). Reflections on the under-researched topic of grief in persons with dementia: A report from a symposium on grief and dementia. American Journal of Hospice and Palliative Care, 27, $567-574$.

Givens, J. L., Prigerson, H. G., Kiely, D. K., Shaffer, M. L., \& Mitchell, S. L. (2011). Grief among family members of nursing home residents with advanced dementia. American Journal of Geriatric Psychiatry, 19, 543-550.

Grand, J. H., Caspar, S., \& Macdonald, S. W. (2011). Clinical features and multidisciplinary approaches to dementia care. Journal of Multidisciplinary Healthcare, 4, 125-147.

Grossman, I., Lutz, M. W., Crenshaw, D. G., Saunders, A. M., Burns, D. K., \& Roses, A. D. (2010). Alzheimer's disease: Diagnostics, prognostics and the road to prevention. The Journal of the European Association for Predictive, Preventive and Personalised Medicine (EPMA), 1, 293-303.

Kiely, D. K., Prigerson, H., \& Mitchell, S. L. (2008). Health care proxy grief symptoms before the death of nursing home residents with advanced dementia. American Journal of Geriatric Psychiatry, 16, 664-673. http://dx.doi.org/10.1097/ JGP.0b013e3181784143 León-Salas, B., Olazará n, J., Muñiz, R., González-Salvador, M. T., \& Martínez-Martín, P. (2011) Caregivers' estimation of patients' quality of life (QoL) in Alzheimer's disease (AD): An approach using the ADRQL. Archives of Gerontology and Geriatrics, 13-18. http://dx.doi.org/10.1016/j.archger.2010.05.021 
Mahoney, R., Regan, C., Katona, C., \& Livingston, G. (2005). Anxiety and depression in family caregivers of people with Alzheimer's disease: The laser-AD study. American Journal of Geriatric Psychiatry, 17, 32-41.

Marwit, S. J., \& Meuser, T. M. (2002). Development and initial validation of an inventory to assess grief in caregivers of persons with Alzheimer's disease. The Gerontologist, 42, 751-765.

Marwit, S. J., \& Meuser, T. M. (2005). Development of a short form inventory to assess grief in caregivers of dementia patients. Death Studies, 29, 191-205.

Maslow, K. (2008). Alzheimer's disease facts and figures. Alzheimer's and Dementia, 4, 110-133.

McLennon, S. M., Bakas, T., Habermann, B., \& Meuser, T. M. (2014). Content and face validity of the Marwit-Meuser Caregiver Grief Inventory (short form) in African American caregivers. Death Studies, 38, 365-373. http://dx.doi.org/10.1080/ 07481187.2013 .766657

Melo, G., Maroco, J., \& de Mendonca, A. (2011). Influence of personality on caregiver's burden, depression and distress related to BPSD. International Journal of Geriatric Psychiatry, 12, 1275-1280.

Meuser, T. M., \& Marwit, S. J. (2001). A comprehensive, stage-sensitive model of grief in dementia caregiving. The Gerontologist, $41,658-670$.

Meuser, T. M., Marwit, S. J., \& Sanders, S. (2004). Assessing grief in family caregivers. In K. Doka (Ed.), Living with grief: Alzheimer's disease (pp. 169-195). Washington, DC: Hospice Foundation of America.

Morris, J. C. (1993). The Clinical Dementia Rating (CDR): Current version and scoring rules. Neurology, 43, $2412-2420$.

Ott, C. H., Sanders, S., \& Kelber, S. T. (2007). Grief and personal growth experience of spouses and adult-child caregivers of individuals with Alzheimer's disease and related dementias. The Gerontologist, 47, 798-809.

Ozarowski, M., Kupsz, J., \& Torlin’s ska, T. (2006). Biological risk factors for Alzheimer's disease. Nowiny Lekarskie, 2, 193-198 (in Polish).

Peterson, B. (2006). Grief and dementia: One caregiver's search for help. Aging Today, 27, 5-13.

Prigerson, H. G., \& Maciejewski, P. K. (2008). Grief and acceptance as opposite sides of the same coin: Setting a research agenda to study peaceful acceptance of loss. British Journal of Psychiatry, 193, 435-437.

Rousson, V., Gasser, T., \& Seifert, B. (2002). Assessing intrarater, interrater and test-retest reliability of continuous measurements. Statistics in Medicine, 21, 3431-3440.

Schulz, R., Boerner, K., Shear, K., Zhang, S., \& Gitlin, L. N. (2006). Predictors of complicated grief among dementia caregivers: A prospective study of bereave-ment. American Journal of Geriatric Psychiatry, 14, 650-658.

Schulz, R., \& Martire, L. M. (2004). Family caregiving of persons with dementia: Prevalence, health effects, and support strategies. American Journal of Geriatric Psychiatry, 12, 240-249.

Schulz, R., McGinnis, K. A., Zhang, S., Martire, L. M., Hebert, R. S., Beach, S. R., et al. (2008). Dementia patient suffering and caregiver depression. Alzheimer's Disease and Associated Disorders, 22, 170-176.

Silverberg, E. (2008). Introducing the 3-A grief intervention model for dementia caregivers: Acknowledge, assess and assist. Omega: Journal of Death \& Dying, 54, 215-235.

Zisook, S., \& Shear, K. (2009). Grief and bereavement: What psychiatrists need to know. World Psychiatry, 8, 67-74.

K. Warchol-Biedermann et al. / Archives of Gerontology and Geriatrics 59 (2014) 462-467 467 